\title{
Alterstice
}

Revue internationale de la recherche interculturelle

International Journal of Intercultural Research

Revista International de la Investigacion Intercultural

\section{Un magnifique congrès 2017 à Antananarivo, et des défis à relever d'ici Genève 2019}

\section{Bureau de l'Association internationale pour la recherche interculturelle (ARIC)}

Volume 7, numéro 1, 2017

URI : https://id.erudit.org/iderudit/1040606ar

DOI : https://doi.org/10.7202/1040606ar

Aller au sommaire du numéro

Éditeur(s)

Alterstice

ISSN

1923-919X (numérique)

Découvrir la revue

Citer ce document

Bureau de l'Association internationale pour la recherche interculturelle (ARIC)

(2017). Un magnifique congrès 2017 à Antananarivo, et des défis à relever d'ici Genève 2019. Alterstice, 7(1), 3-5. https://doi.org/10.7202/1040606ar

Droits d'auteur (C) Bureau de l'Association internationale pour la recherche interculturelle (ARIC), 2017
Ce document est protégé par la loi sur le droit d'auteur. L'utilisation des services d'Érudit (y compris la reproduction) est assujettie à sa politique d'utilisation que vous pouvez consulter en ligne.

https://apropos.erudit.org/fr/usagers/politique-dutilisation/ 


\title{
Un magnifique congrès 2017 à Antananarivo, et des défis à relever d'ici Genève 2019
}

\author{
Bureau de l'Association internationale pour la recherche interculturelle (ARIC) ${ }^{1}$
}

Du 23 au 27 mai dernier, l'ARIC a vécu son $16^{\mathrm{e}}$ congrès, qui s'est déroulé pour la première fois de son histoire dans la zone de l'Océan indien, à Antananarivo (Madagascar). Magnifiquement accueilli par le Centre de Recherche et d'Étude sur les Constructions Identitaires (CRECI) de l'Université d'Antananarivo, avec un comité d'organisation enthousiaste et dévoué autour de sa présidente Dominique Tiana Razafindratsimba, ce congrès a été l'occasion d'échanges fructueux entre chercheurs, praticiens, étudiants et membres de la société civile, sur la thématique Nouveaux contextes et nouvelles pratiques interculturelles. Globalisation et circulation. Durant quatre jours, conférences, tables rondes, symposiums et communications ont suscité de riches débats, et permis notamment la construction de ponts entre chercheurs malgaches et chercheurs venus des autres pays. Dans des conditions parfois difficiles, le comité d'organisation a su à la fois travailler avec rigueur pour un congrès d'une grande qualité scientifique et accueillir les membres de I'ARIC et autres congressistes avec chaleur, flexibilité et hospitalité. Outre la partie scientifique, les 170 participants au congrès ont pu découvrir la richesse et la diversité de la culture malgache, sur le site même du congrès mais aussi lors d'une soirée culturelle, de la soirée de gala et d'une sortie organisée en conclusion du congrès. Avec ses moments interculturels d'une qualité exceptionnelle, ce congrès 2017 a, à la fois, impressionné, touché et profondément transformé les participants, et s'est avéré une contribution majeure à l'atteinte d'un des objectifs de I'ARIC, soit de renforcer les collaborations authentiques et réciproques Nord-Sud.

Le congrès de l'ARIC, qui se déroule tous les deux ans et accueille l'assemblée générale de l'association, est aussi l'occasion d'un bilan d'activités de la période écoulée. En l'occurrence, les 21 mois séparant le congrès d'Antananarivo de celui de Strasbourg en 2015 ont été bien remplis. Même s'ils n'ont pas permis de répondre à tous les souhaits de développement de l'ARIC, ces deux ans ont permis d'atteindre globalement les objectifs fixés en 2015, qui étaient : 1) améliorer la communication et la vitrine de l'association pour accueillir de nouveaux membres et répondre aux besoins de toutes et de tous, 2) poursuivre le développement des liens Nord-Sud et le développement de l'association dans les pays où elle est moins présente, dans une perspective de reconnaissance et d'échange des savoirs interculturels multiples et diversifiés, 3) participer au développement de la recherche interculturelle sous ses diverses formes, disciplines et méthodologies et dans des domaines multiples et innovants, 4) renforcer les interfaces entre chercheurs et professionnels du domaine interculturel, 5) favoriser la relève et le mentorat auprès des jeunes chercheurs et professionnels du domaine interculturel, 6) développer les réseaux internationaux et transnationaux par le biais de publications et de projets menés par des membres de l'ARIC dans leurs sociétés et institutions et enfin 7) assurer un financement pérenne à l'association. La période écoulée a aussi été marquée par le colloque d’Olinda au Brésil en avril 2016, lors de l'année intermédiaire entre les deux congrès. Nous avons eu l'occasion, dans la Rubriqu'ARIC 6(1), de vous parler de ce colloque, qui a été une grande réussite, avec quelque 300 participants et 150 travaux présentés.

Concernant notre objectif de communication, un nouveau site internet a été développé et mis en ligne récemment (http://www.aric-interculturel.com/), permettant à l'ARIC de disposer désormais de son propre outil (l'ancien site était hébergé sur le site de l'université de Fribourg). Ce nouveau site peut être désormais enrichi et alimenté de manière plus flexible et réactive. Une lettre d'information est également envoyée mensuellement aux membres de I'ARIC, favorisant la transmission d'information au sujet des publications, congrès et colloques pertinents et de divers sujets transmis par les membres. L'ARIC dispose en outre à présent d'une rubrique dans la revue Alterstice - la Rubriqu'ARIC que vous êtes en train de lire - ce qui permet également de rendre compte des nouveautés de l'association et des contenus qui s'y développent. Enfin, une chaîne YouTube de l'ARIC a été créée, grâce au travail de Ourakoye Bembello, stagiaire de maîtrise en médiation interculturelle auprès de l'ARIC 
durant quelques mois en 2016. Cette chaîne offre notamment des vidéos d'entrevues avec des spécialistes de la recherche interculturelle et la vidéo d'une table ronde tenue lors du colloque d'Olinda (les liens vers ces vidéos sont disponibles sur le site de l'ARIC : http://www.aric-interculturel.com/photosvideos/).

À propos des liens Nord-Sud, le fait que le congrès 2017 se soit tenu à Madagascar contribue à la stratégie de déploiement de l'ARIC dans des contextes où elle n'était pas encore présente. Tenir un congrès si loin des bases habituelles de I'ARIC représentait certains risques, entre autres celui de ne pas attirer suffisamment de participants, particulièrement parmi les membres de I'ARIC. Le défi a toutefois été parfaitement relevé, et ce congrès a permis la construction de liens forts entre I'ARIC et l'université d'Antananarivo, qu'il s'agira de poursuivre et maintenir lors des activités et congrès à venir. En se déroulant à Genève en juin 2019, le prochain congrès, par un retour à un contexte plus traditionnel, permettra l'alternance indispensable pour que soient satisfaits les différents membres de l'association.

L'objectif visant l'innovation et la diversité s'est notamment traduit par l'instauration, lors du colloque d'Olinda, d'une thématique portant sur les réseaux, transnationaux ou virtuels, ce qui a permis un nouveau champ de développement de la recherche interculturelle à I'ARIC, caractérisé par la multidisciplinarité. Les thématiques des nouvelles technologies de l'information et des réseaux transnationaux ont ensuite été reprises lors du congrès d'Antananarivo, qui a aussi été l'occasion de traiter de nouvelles préoccupations comme l'art et la littérature dans une perspective interculturelle.

Concernant les interfaces entre recherche et pratique, l'ARIC vise à ce que les informations échangées, tant par la lettre d'information aux membres que par le site internet ou les entrevues vidéo insérées sur la chaine YouTube, concernent tant le monde de la recherche interculturelle que celui de l'intervention et des pratiques interculturelles, les deux se nourrissant mutuellement. Les praticiens sont en outre toujours les bienvenues lors des congrès et colloques de I'ARIC. Bon nombre ont d'ailleurs participé - et certains ayant même offert une communication - lors du congrès d'Antananarivo. Ce congrès 2017 a aussi été l'occasion d'un forum Femmes et féminismes à Madagascar, organisé en paracongrès et ouvert gratuitement aux membres et organismes de la société civile, toujours dans cette perspective de décloisonnement, d'échange de savoirs et d'enrichissement réciproque.

L'objectif concernant la relève n'a pas été oublié. Le prix ARIC de la meilleure thèse en recherche interculturelle a vécu sa troisième édition à l'occasion du congrès d'Antananarivo. Une vingtaine de candidatures ont été soumises et la lauréate du prix 2017, Mme Audrey Heine, a eu l'occasion de présenter lors d'une conférence plénière sa brillante thèse intitulée Entre pays d'origine et pays d'accueil. Les enjeux identitaires de la (non) reconnaissance des descendant-e-s d'immigré-es au pays d'origine. De jeunes chercheurs et étudiants sont en outre régulièrement présents lors des congrès et colloques de I'ARIC, plusieurs participant également comme bénévoles. La cotisation réduite de l'ARIC pour les étudiants vise aussi à ce que ces jeunes chercheurs et étudiant puissent continuer à fréquenter l'association et l'enrichir de leurs perspectives souvent innovantes.

Quant aux publications et réseaux de recherche, ces deux dernières années ont vu la publication d'ouvrages issus du colloque d'Olinda (2016), du congrès de Strasbourg (2015) et du colloque de Sfax (2014). Ces publications montrent la vitalité internationale des connaissances en recherche interculturelle favorisées par l'ARIC, ses rencontres et ses réseaux. Soutenue par I'ARIC, la revue Alterstice, avec toute son indépendance éditoriale, continue également à diffuser des articles de recherche internationaux portant sur la recherche interculturelle. Les numéros thématiques correspondent au développement de réseaux de chercheurs. La revue est par ailleurs à présent référencée sur la plateforme scientifique Érudit, qui favorise la diffusion, la préservation numérique et la visibilité en libre-accès depuis 18 ans des publications scientifiques et culturelles en sciences humaines et sociales. Ce travail de promotion et de référencement de la revue contribue à faire connaître les travaux menés dans le champ de l'interculturel au niveau international. Plusieurs réseaux de recherche ont en outre émergé des congrès et colloques, aboutissant à des études et à des publications internationales (femmes et féminismes en dialogue, médiations interculturelles, familles immigrantes et école, dynamiques transnationales et citoyenneté, immigration et handicap, nouvelles technologies de l'information et interculturel, etc.).

Le dernier objectif, celui visant à assurer un financement pérenne à l'association, n'a en revanche pas pu être atteint. Certes, des décisions ont été prises lors de la dernière assemblée générale, comme une légère augmentation des cotisations, mais I'ARIC vit actuellement sur ses réserves, et l'objectif de financement pérenne a donc été défini comme la priorité du nouveau Bureau et du nouveau Conseil élus lors du congrès d'Antananarivo. Ce point nous amène ainsi aux trois grands objectifs de l'ARIC pour les deux années à venir, soit d'ici au congrès de Genève en juin 2019 : 1) assurer de nouveaux financements à l'ARIC et une stratégie de 
financement récurrente et solide, 2) développer de nouveaux axes de travail et de recherche transversaux, lors des congrès et colloques mais également entre ceux-ci, avec des personnes de I'ARIC responsables de ces différents axes et 3) continuer à développer, favoriser et renforcer la relève de l'ARIC. Ces objectifs se situent ainsi dans la droite ligne de ceux de 2015-2017, se centrant sur la poursuite, le développement et le renforcement d’objectifs déjà amorcés. Il faut rappeler également notre volonté de toujours favoriser, de manière transversale, la multi- voire l'inter-disciplinarité, les collaborations Nord-Sud et les échanges entre recherche et pratique.

Nous ne pouvons conclure cette Rubriqu'ARIC sans revenir sur le formidable congrès 2017 que nous venons de vivre à Madagascar. Le congrès d'Antananarivo a été un extraordinaire incubateur et une immense source d'énergie pour les participants, mais aussi plus largement pour l'ARIC dans son ensemble, son Bureau et son Conseil. Concernant I'ARIC, les réussites mises en avant témoignent du dynamisme et de la richesse de la vie scientifique de l'association, mais les points relevés montrent également l'importance des défis à venir. La richesse des réflexions et des débats suscités lors du congrès d'Antananarivo à propos des questions de mondialisation et de circulation, parallèlement à l'actualité qui ne cesse de nous interpeller sur ces sujets, témoigne du rôle essentiel de la recherche interculturelle et d'une association comme l'ARIC. Dès lors, que l'énergie formidable issue de ce congrès et de l'accueil reçu de nos hôtes malgaches puisse se propager dans les activités et réflexions de l'ARIC et de ses membres d'ici à son prochain congrès!

Bien interculturellement,

Le Bureau de I'ARIC 2017-2019 : Michèle Vatz Laaroussi, présidente, Elaine Costa Fernandez, vice-présidente, Alida Gulfi, trésorière, Melissa Arneton, vice-trésorière, Xavier Conus, secrétaire administratif, Danielle Lezou Koffi, secrétaire générale.

\section{Rattachement des auteurs}

Bureau de I'ARIC

\section{Correspondance}

aric_assoc@yahoo.fr

\section{Pour citer cet article}

Bureau de l'ARIC. (2017). Un magnifique congrès 2017 à Antananarivo, et des défis à relever d'ici Genève 2019 [Rubriqu'ARIC]. Alterstice, 7(1), 3-6. 